Tidal dynamics in cosmological spacetimes

This article has been downloaded from IOPscience. Please scroll down to see the full text article.

2007 Class. Quantum Grav. 245031

(http://iopscience.iop.org/0264-9381/24/20/008)

View the table of contents for this issue, or go to the journal homepage for more

Download details:

IP Address: 128.206.162.204

The article was downloaded on 17/08/2010 at 17:22

Please note that terms and conditions apply. 


\title{
Tidal dynamics in cosmological spacetimes
}

\author{
Bahram Mashhoon $^{1}$, Nader Mobed ${ }^{2}$ and Dinesh Singh ${ }^{2}$ \\ ${ }^{1}$ Department of Physics and Astronomy, University of Missouri-Columbia, Columbia, \\ MO 65211, USA \\ ${ }^{2}$ Department of Physics, University of Regina, Regina, Saskatchewan, S4S 0A2, Canada \\ E-mail: mashhoonb@missouri.edu,nader.mobed@uregina.ca and singhd@uregina.ca
}

Received 9 May 2007, in final form 19 July 2007

Published 2 October 2007

Online at stacks.iop.org/CQG/24/5031

\begin{abstract}
We study the relative motion of nearby free test particles in cosmological spacetimes, such as the FLRW and LTB models. In particular, the influence of spatial inhomogeneities on local tidal accelerations is investigated by means of simple spherically symmetric models. The implications of our results for the dynamics of the solar system are briefly discussed. That is, on the basis of the models studied in this paper, we estimate the tidal influence of the cosmic gravitational field on the orbit of the Earth around the Sun and show that the corresponding temporal rate of variation of the astronomical unit is negligibly small.
\end{abstract}

PACS number: $04.20 . \mathrm{CV}$

\section{Introduction}

The gravitational influence of distant galaxies extends over all of the bodies in our cosmic neighbourhood; therefore, the relative motion of these bodies would be affected by the tidal field of distant masses. The local tidal effects of distant cosmic masses as a whole can be studied by means of general relativistic cosmological models. Hence we consider-for the sake of concreteness - tidal dynamics in the standard homogeneous FLRW models as well as certain rather simple spherically symmetric inhomogeneous models. Inhomogeneous cosmological models are of current interest as they may be potentially useful in solving the dark-energy problem; however, we emphasize that our simple models are employed here merely to illustrate our general approach.

To study the lowest order tidal effects invariantly, it is necessary to choose an observer and determine the projection of the Riemann tensor on the observer's local frame; these curvature components are then employed to define a quasi-inertial system of normal coordinates in the neighbourhood of the observer's worldline. The motion of test bodies can be studied in such a Fermi coordinate system in accordance with the ideas and methods developed in detail in 
[1]. In the cosmological models that we examine, it is natural to choose the fundamental observers-i.e. those that are spatially at rest-as fiducial observers and study cosmological tidal effects in their immediate neighbourhoods. A general outline of the standard procedures for the study of tidal dynamics is provided below.

In general relativity, it is natural to study the relative motion of a test particle with respect to an observer in the quasi-inertial Fermi coordinate system [1] established about the worldline of the observer. To simplify matters, let us assume that the observer follows a geodesic worldline $\mathcal{C}$ and let $\lambda^{\mu}{ }_{(\alpha)}$ be the observer's local orthonormal tetrad frame that is parallel transported along $\mathcal{C}$. Here $\lambda^{\mu}{ }_{(0)}=\mathrm{d} x^{\mu} / \mathrm{d} \tau$ is the observer's local temporal axis and $\tau$ is its proper time, while $\lambda^{\mu}{ }_{(i)}, i=1,2,3$, are unit gyro directions that constitute the observer's local spatial frame. We choose units such that $c=1$, unless specified otherwise. An event $P$ in the neighbourhood of the observer has Fermi coordinates $X^{\mu}=(T, X)$; in fact, $P$ can be orthogonally connected to $\mathcal{C}$ at $P_{0}$ via a unique spacelike geodesic of proper length $\sigma$ such that $\sigma=0$ at $P_{0}$. Let the proper time along $\mathcal{C}$ at $P_{0}$ be $\tau$ and $\xi^{\mu}=\left(\mathrm{d} x^{\mu} / \mathrm{d} \sigma\right)_{0}$ be the unit tangent vector to the spacelike geodesic at $P_{0}$; then the Fermi coordinates of the event $P$ are defined by $T=\tau$ and $X^{i}=\sigma \xi^{\mu} \lambda_{\mu}{ }^{(i)}$. It follows that the observer permanently occupies the spatial origin of this Fermi coordinate system.

The spacetime metric in Fermi coordinates can be expressed as

$$
\begin{aligned}
& { }^{F} g_{00}=-1-{ }^{F} R_{0 i 0 j}(T) X^{i} X^{j}+\cdots, \\
& { }^{F} g_{0 i}=-\frac{2}{3}{ }^{F} R_{0 j i k}(T) X^{j} X^{k}+\cdots, \\
& { }^{F} g_{i j}=\delta_{i j}-\frac{1}{3}{ }^{F} R_{i k j l}(T) X^{k} X^{l}+\cdots,
\end{aligned}
$$

where

$$
{ }^{F} R_{\alpha \beta \gamma \delta}(T)=R_{\mu \nu \rho \sigma} \lambda^{\mu}{ }_{(\alpha)} \lambda^{\nu}{ }_{(\beta)} \lambda^{\rho}{ }_{(\gamma)} \lambda^{\sigma}{ }_{(\delta)}
$$

is the Riemann curvature tensor along $\mathcal{C}$ projected on the tetrad frame of the observer. The Fermi coordinates are admissible in a cylindrical spacetime region around $\mathcal{C}$ with $|\boldsymbol{X}|<\mathcal{L}$, where $\mathcal{L}$ is a measure of the radius of curvature of spacetime.

The motion of a free test particle is given by the geodesic equation

$$
\frac{\mathrm{d}^{2} X^{\mu}}{\mathrm{d} s^{2}}+{ }^{F} \Gamma_{\alpha \beta}^{\mu} \frac{\mathrm{d} X^{\alpha}}{\mathrm{d} s} \frac{\mathrm{d} X^{\beta}}{\mathrm{d} s}=0
$$

where $\mathrm{d} X^{\mu} / \mathrm{d} s=\Gamma(1, \boldsymbol{V})$ is the particle's timelike 4-velocity vector. Equation (5) can be expressed as

$$
\frac{1}{\Gamma} \frac{\mathrm{d} \Gamma}{\mathrm{d} T}=-{ }^{F} \Gamma_{\alpha \beta}^{0} \frac{\mathrm{d} X^{\alpha}}{\mathrm{d} T} \frac{\mathrm{d} X^{\beta}}{\mathrm{d} T}
$$

and the reduced geodesic equation

$$
\frac{\mathrm{d}^{2} X^{i}}{\mathrm{~d} T^{2}}+\left({ }^{F} \Gamma_{\alpha \beta}^{i}-{ }^{F} \Gamma_{\alpha \beta}^{0} V^{i}\right) \frac{\mathrm{d} X^{\alpha}}{\mathrm{d} T} \frac{\mathrm{d} X^{\beta}}{\mathrm{d} T}=0 .
$$

This latter equation, to linear order in distance away from $\mathcal{C}$, is the generalized Jacobi equation [2]

$$
\begin{aligned}
\frac{\mathrm{d}^{2} X^{i}}{\mathrm{~d} T^{2}}+{ }^{F} R_{0 i 0 j} & X^{j}+2{ }^{F} R_{i k j 0} V^{k} X^{j} \\
& +\frac{2}{3}\left(3^{F} R_{0 k j 0} V^{i} V^{k}+{ }^{F} R_{i k j l} V^{k} V^{l}+{ }^{F} R_{0 k j l} V^{i} V^{k} V^{l}\right) X^{j}=0
\end{aligned}
$$


and the modified Lorentz factor is given by

$$
\frac{1}{\Gamma^{2}}=1-V^{2}+{ }^{F} R_{0 i 0 j} X^{i} X^{j}+\frac{4}{3}{ }^{F} R_{0 j i k} X^{j} V^{i} X^{k}+\frac{1}{3}{ }^{F} R_{i k j l} V^{i} X^{k} V^{j} X^{l} .
$$

Equation (8) reduces to the Jacobi equation when the velocity-dependent terms are negligibly small.

In some situations of physical interest, it is possible to have purely one-dimensional motion, say along the $Z$-direction. In this case, equation (8) reduces to

$$
\frac{\mathrm{d}^{2} Z}{\mathrm{~d} T^{2}}+k(T)\left(1-2 \dot{Z}^{2}\right) Z=0
$$

where $\dot{Z}=\mathrm{d} Z / \mathrm{d} T$ and $k(T)={ }^{F} R_{T Z T Z}$. For $|\dot{Z}| \ll 1$, equation (10) reduces to the standard Jacobi equation. However, when the relative speed cannot be neglected in comparison with the speed of light, the generalized Jacobi equation (10) has solutions with $\dot{Z}= \pm 1 / \sqrt{2}$ such that the relative motion within the linear approximation scheme is uniform at the critical speed $V_{c}=1 / \sqrt{2}$. Below this speed, the relative motion should essentially conform to expectations based on the post-Newtonian approximation. On the other hand, novel relativistic tidal effects are expected to occur above the critical speed.

Let the critical solutions of equation (10) be expressed as $Z_{c}(T)=Z_{i} \pm V_{c}\left(T-T_{i}\right)$, where $T_{i}$ is an initial time and $Z_{i}=Z_{c}\left(T_{i}\right)$. Then, the behaviour of $Z(T)$ near a critical solution can be examined by introducing $\zeta(T)=Z-Z_{c}$. It follows from (10) that

$$
\frac{\mathrm{d}^{2} \zeta}{\mathrm{d} T^{2}}-2 k(T)\left(\dot{\zeta}^{2} \pm 2 V_{c} \dot{\zeta}\right)\left(\zeta+Z_{c}\right)=0
$$

Keeping only terms of linear order in (11), we find

$$
\frac{1}{\dot{\zeta}} \frac{\mathrm{d} \dot{\zeta}}{\mathrm{d} T}= \pm 4 V_{c} Z_{c} k(T)
$$

where $\dot{\zeta}=\mathrm{d} \zeta / \mathrm{d} T$. It follows that the long-term behaviour of this motion can be determined from

$$
\mathcal{I}=\int_{T_{i}}^{\infty}\left(T+C_{i}\right) k(T) \mathrm{d} T,
$$

where $C_{i}=-T_{i} \pm \sqrt{2} Z_{i}$ is a constant and $\pm 2 V_{c} Z_{c}=T+C_{i}$. For instance, the critical solutions are attractors for $\mathcal{I}=-\infty$ [2]. Equation (10) has been extensively studied in black hole spacetimes in connection with the problem of astrophysical jets [3, 4].

The main purpose of this paper is to study tidal dynamics in some cosmological models. It turns out that for the standard FLRW models, $k(T)$ is characterized by the deceleration of the universe. This point is demonstrated in the following section. In section 3 , a locally inhomogeneous model is employed to determine the corresponding $k(T)$. In this way, the influence of cosmic spatial inhomogeneities on local measurements is elucidated. This treatment is extended in section 4 to the Lemaitre-Tolman-Bondi (LTB) models. The consequences of the results of section 4 for the dynamics of the solar system are briefly discussed in section 5 and in the appendix. Section 6 contains a brief discussion of our results.

\section{FLRW models}

Let us first consider the FLRW spacetimes given in isotropic coordinates $x^{\mu}=\left(t, x^{i}\right)$ by

$$
-\mathrm{d} s^{2}=-\mathrm{d} t^{2}+\frac{S^{2}(t)}{f^{2}(r)} \delta_{i j} \mathrm{~d} x^{i} \mathrm{~d} x^{j},
$$


where $S(t)>0$ is the scale factor and

$$
f(r)=1+\frac{1}{4} \kappa r^{2}
$$

with $\kappa=-1,0$, or +1 for the open, flat, or closed universe models, respectively. We note that the spatial coordinates $x^{i}$ are dimensionless, while the scale factor $S$ has the dimension of length.

A fundamental observer in this spacetime occupies a fixed position in space and follows a geodesic worldline $\mathcal{C}$ with proper time $\tau=t$. Let us choose a fundamental observer with $\lambda^{\mu}{ }_{(0)}=\delta^{\mu}{ }_{0}$ and $\lambda^{\mu}{ }_{(i)}=[f(r) / S(t)] \delta^{\mu}{ }_{i}$, which can be shown to be parallel transported along the geodesic worldline. The curvature tensor ${ }^{F} R_{\alpha \beta \gamma \delta}$ along $\mathcal{C}$ can be expressed as a $6 \times$ 6 matrix $\left({ }^{F} \mathcal{R}_{J K}\right)$, where the indices $J$ and $K$ range over the set $\{01,02,03,23,31,12\}$. Then,

$$
{ }^{F} \mathcal{R}=\left(\begin{array}{cc}
E & B \\
B^{\dagger} & N
\end{array}\right)
$$

where for the FLRW models $E=u(t) I, B=0$, and $N=w(t) I$. Here, $I$ is the $3 \times 3$ identity matrix and

$$
\begin{aligned}
& u(t)=-\frac{1}{S} \frac{\mathrm{d}^{2} S}{\mathrm{~d} t^{2}} \\
& w(t)=\frac{1}{S^{2}}\left(\frac{\mathrm{d} S}{\mathrm{~d} t}\right)^{2}+\frac{\kappa}{S^{2}}
\end{aligned}
$$

We note in passing that (17) and (18) imply

$$
S \frac{\mathrm{d} w}{\mathrm{~d} t}=-2(u+w) \frac{\mathrm{d} S}{\mathrm{~d} t} .
$$

It is possible to show that the only nonzero components of the Einstein tensor, $G_{\mu \nu}=R_{\mu \nu}-\frac{1}{2} g_{\mu \nu} R$, are given by

$$
\begin{aligned}
& G_{\mu \nu} \lambda^{\mu}{ }_{(0)} \lambda^{\nu}{ }_{(0)}=3 w(t), \\
& G_{\mu \nu} \lambda^{\mu}{ }_{(i)} \lambda^{\nu}{ }_{(j)}=[2 u(t)-w(t)] \delta_{i j} .
\end{aligned}
$$

The gravitational field equations (with a cosmological constant $\Lambda$ ) are given by

$$
G_{\mu \nu}+\Lambda g_{\mu \nu}=8 \pi G T_{\mu \nu},
$$

where $T_{\mu \nu}$ is the stress-energy tensor for a perfect fluid

$$
T_{\mu \nu}=\mu(t) u_{\mu} u_{v}+p(t)\left(g_{\mu \nu}+u_{\mu} u_{v}\right) .
$$

Here, $\mu(t)$ and $p(t)$ are the invariant density and pressure of the cosmic fluid, and the fundamental observers are assumed to be comoving with the cosmic fluid, i.e. $u^{\mu}=\lambda^{\mu}{ }_{(0)}$. Equations (20)-(23) imply that $u$ and $w$, defined respectively by (17) and (18), are given by

$$
\begin{aligned}
& u(t)=\frac{4 \pi G}{3}(\mu+3 p)-\frac{\Lambda}{3}, \\
& w(t)=\frac{8 \pi G}{3} \mu+\frac{\Lambda}{3} .
\end{aligned}
$$

It follows from (19), (24), (25), and the constancy of $\Lambda$ that

$$
\frac{1}{3} \frac{\mathrm{d} \mu}{\mathrm{d} t}=-\frac{1}{S} \frac{\mathrm{d} S}{\mathrm{~d} t}(\mu+p)
$$


This is the expression of the first law of thermodynamics for the adiabatic flow of the perfect fluid under consideration here. The standard Hubble and deceleration parameters $(H$ and $q)$ are given by

$$
H=\frac{1}{S} \frac{\mathrm{d} S}{\mathrm{~d} t}, \quad q H^{2}=-\frac{1}{S} \frac{\mathrm{d}^{2} S}{\mathrm{~d} t^{2}}
$$

so that $u=q H^{2}$ and $w=H^{2}+\kappa / S^{2}$.

A Fermi coordinate system $(T, \boldsymbol{X})$ can be established along the worldline of a fundamental observer; then, the observer has Fermi coordinates $(T, \boldsymbol{0})$, where $T=t$. The FLRW spacetime metric in spherical Fermi coordinates

$$
X=\rho \sin \Theta \cos \Phi, \quad Y=\rho \sin \Theta \sin \Phi, \quad Z=\rho \cos \Theta,
$$

can be expressed as [5]

$-\mathrm{d} s^{2}=-\left[1+u(T) \rho^{2}\right] \mathrm{d} T^{2}+\mathrm{d} \rho^{2}+\left[1-\frac{1}{3} w(T) \rho^{2}\right] \rho^{2}\left(\mathrm{~d} \Theta^{2}+\sin ^{2} \Theta \mathrm{d} \Phi^{2}\right)$.

This is based on (1)-(3) and thus holds only to second order in distance away from the observer. It appears that explicit Fermi coordinates can be constructed for FLRW models based on the results of [4]; however, the scale factor $S(t)$ must then be explicitly specified. To have a general treatment for arbitrary $S(t)$, we must use the implicit approach as in (1)-(3).

The spatial isotropy of the FLRW models implies that an equation of motion of the form of equation (10) is possible along any direction in space with $k(T)=u(T)=q H^{2}$. Thus the nature of such motion is characterized by the sign of the deceleration parameter. Imagine, for instance, a free test particle moving radially away from the observer. For an initially infrarelativistic particle with recession speed less than $V_{c}$, the particle decelerates (accelerates) for $q>0(q<0)$, as expected. However, for an initially ultrarelativistic particle with recession speed above $V_{c}$, the particle accelerates (decelerates) for $q>0(q<0)$. In the following sections, we simply concentrate on the nature of $k(T)$ in certain inhomogeneous models.

In the slow-motion approximation, it is possible to extract a 'Newtonian' gravitational potential [5]

$$
\mathcal{V}_{\mathrm{N}}=\frac{1}{2} q H^{2} \rho^{2}
$$

from (29). Equation (30) has been employed in the discussion of the influence of cosmology on local phenomena [6]. Reference [6] contains only a partial list of papers on this subject; for background material and further references, see [7]. The form of the quadratic potential (30) suggests that the influence of the cosmic gravitational field is reflected in local experiments - in the solar system, for instance-via a relative tidal acceleration of the form $\boldsymbol{g}_{\text {cosmos }}=-q H^{2} \boldsymbol{X}$. It is interesting to note that the deceleration parameter $q$ also appears in the second-order expansion of luminosity distance $d_{L}$ versus redshift $z$, that is

$$
d_{L}=\frac{1}{H} z+\frac{1}{2 H}(1-q) z^{2}+\cdots
$$

This 'degeneracy' is removed by spatial inhomogeneities as demonstrated in the following section.

It appears highly likely that instead of providing evidence for dark energy, the observational data from type Ia supernovae have demonstrated the inadequacy of the standard spatially homogeneous FLRW models of the universe. That is, spatial inhomogeneities must be taken into account when comparing observational data with theoretical models of the universe. This is based on the fact that in the luminosity distance-redshift relation, spatial inhomogeneities can mimic dark energy [8]. It is therefore important to develop 
inhomogeneous models that could account for the current observational data in cosmology; this is an active area of current research and a recent useful review of this topic is contained in [9].

\section{Inhomogeneous models}

It is important to investigate how spatial inhomogeneities in the universe are reflected in local spacetime measurements. We begin with a metric of the form $[5,10]$

$$
-\mathrm{d} s^{2}=-a^{2}(t, r) \mathrm{d} t^{2}+b^{2}(t, r) \mathrm{d} r^{2}+\mathcal{R}^{2}(t, r)\left(\mathrm{d} \theta^{2}+\sin ^{2} \theta \mathrm{d} \phi^{2}\right),
$$

where

$$
\begin{aligned}
& a(t, r)=1+\frac{1}{2} \alpha(t) r^{2}+\cdots \\
& b(t, r)=\left[1+\frac{1}{2} \beta(t) r^{2}+\cdots\right] S(t), \\
& \mathcal{R}(t, r)=r\left[1+\frac{1}{2} \gamma(t) r^{2}+\cdots\right] S(t) .
\end{aligned}
$$

Here, $\alpha, \beta$ and $\gamma$ are parameters in the expansion of the cosmic gravitational potentials in powers of the dimensionless radial coordinate $r$ away from the centre of spherical symmetry $(r=0)$. Expansions (33)-(35) are valid when the cosmological redshift $z$ is sufficiently small compared to unity; hence, such a 'local' model cannot be used over large cosmological scales. This model has been discussed at length [5, 10]; in particular, it has been shown in [10] that this 'local' inhomogeneous model is generally valid for a cosmic fluid that has pressure and satisfies a reasonable equation of state of the form $p=p(\mu)$ with $p \geqslant 0, \mu \geqslant 0$, and $\mu \geqslant 3 p$ or $\mu \geqslant p$. We note that at the order of approximation indicated in (33)-(35), the homogeneous models of the previous section are recovered for $\alpha=0$ and $\beta=\gamma=-\kappa / 2$.

We imagine, as before, observers that are fixed in space and carry orthonormal tetrad frames along their worldlines. The natural tetrad of such an observer is diagonal with nonzero elements $\lambda^{0}{ }_{(0)}=a^{-1}, \lambda^{1}{ }_{(1)}=b^{-1}, \lambda^{2}{ }_{(2)}=\mathcal{R}^{-1}$, and $\lambda^{3}{ }_{(3)}=(\mathcal{R} \sin \theta)^{-1}$. We are interested in a Fermi coordinate system along the worldline $\mathcal{C}$ of the observer that is at the centre of spherical symmetry. It can be shown explicitly using equations (32)-(35) that $\mathcal{C}$ is a geodesic and the spatial frame is indeed parallel propagated along $\mathcal{C}$ in the $r \rightarrow 0$ limit. Alternatively, one can employ local Cartesian coordinates as in the previous section. In any case, the curvature components ${ }^{F} R_{\alpha \beta \gamma \delta}$ along $\mathcal{C}$ can be explicitly calculated and the results can be expressed as a $6 \times 6$ matrix (16) with

$$
E=U(t) I, \quad B=0, \quad N=W(t) I,
$$

where for this inhomogeneous model

$$
\begin{aligned}
& U(t)=q H^{2}+\frac{\alpha(t)}{S^{2}}, \\
& W(t)=H^{2}+\frac{\beta(t)-3 \gamma(t)}{S^{2}} .
\end{aligned}
$$

Here, $q$ and $H$ are defined in terms of the scale factor $S(t)$ as in (27). Moreover, the analogues of equations (24) and (25) hold for $\mu(t, r)$ and $p(t, r)$ evaluated at $r=0$; that is,

$$
\begin{aligned}
& U(t)=\frac{4 \pi G}{3}[\mu(t, 0)+3 p(t, 0)]-\frac{\Lambda}{3}, \\
& W(t)=\frac{8 \pi G}{3} \mu(t, 0)+\frac{\Lambda}{3} .
\end{aligned}
$$


The spacetime metric in spherical Fermi coordinates about $\mathcal{C}$ has the same form as equation (29), except that $u(t) \rightarrow U(t)$ and $w(t) \rightarrow W(t)$; moreover, $t=T$, since $r=0$ along $\mathcal{C}$. Let us note in this connection that the quantity $\beta-3 \gamma$ in (38) is related to the spatial curvature and reduces to $\kappa$ in the homogeneous limit $[5,10]$. The motion of a free test particle in the neighbourhood of $\mathcal{C}$ can be studied as in the previous section except that in this case $k(T)=U(T)$ in equation (10). Furthermore, the influence of the cosmic gravitational field on local physics in the slow-motion approximation appears as a 'Newtonian' acceleration of the form

$$
\boldsymbol{g}_{\mathrm{cosmos}}=-H^{2}(q-C) \boldsymbol{X},
$$

where $C(t)$ is the inhomogeneity parameter given by

$$
C=-\frac{\alpha}{(S H)^{2}} \text {. }
$$

It is interesting to note that in this inhomogeneous model, the luminosity distance-redshift relation can be expressed as $[5,10]$

$$
d_{L}=\frac{1}{H} z+\frac{1}{2 H}[1-(q+C)] z^{2}+\cdots,
$$

so that the measured 'deceleration' parameter is in fact $q+C$. It follows that both $q$ and $C$ could be determined from observation if it were possible to measure $k(T)=H^{2}(q-C)$ as well. It follows from (39) that

$$
H^{2}(q-C)=4 \pi G\left[\frac{1}{3} \mu(t, 0)+p(t, 0)\right]-\frac{\Lambda}{3} .
$$

Hence, in the absence of a cosmological constant we have $C<q$, which is a noteworthy inequality,

Finally, let us note that by imposing the comoving coordinate condition [10]

$$
\frac{\mathrm{d}}{\mathrm{d} t}(\beta-3 \gamma)=-2 H(t) \alpha(t),
$$

one can show from (39) and (40) that

$$
\frac{\partial}{\partial t} \mu(t, 0)=-3 H(t)[\mu(t, 0)+p(t, 0)],
$$

which is the analogue of (26) in this inhomogeneous case.

\section{LTB models}

Imagine a spacetime metric of the form (32) with

$$
a(t, r)=1, \quad b(t, r)=\frac{\mathcal{R}^{\prime}(t, r)}{\sqrt{1+2 \mathcal{E}(r)}},
$$

where $\mathcal{R}^{\prime}=\partial \mathcal{R} / \partial r$. It turns out that the gravitational field equations (22) are satisfied in this case for pure dust

$$
T_{\mu \nu}=\mu(t, r) u_{\mu} u_{\nu}
$$

in comoving coordinates (i.e. $u^{\mu}=\delta^{\mu}{ }_{0}$ ) provided

$$
\begin{aligned}
& \mathcal{E}(r)=\frac{1}{2} \dot{\mathcal{R}}^{2}-\frac{G M(r)}{\mathcal{R}}-\frac{\Lambda}{6} \mathcal{R}^{2}, \\
& \frac{\mathrm{d} M(r)}{\mathrm{d} r}=4 \pi \mu(t, r) \mathcal{R}^{2} \mathcal{R}^{\prime} .
\end{aligned}
$$


Here, $\mathcal{E}(r)$ with $\mathcal{E}>-1 / 2$ has the interpretation of the net energy per unit mass of the spherical shell of dust at radius $r, M(r)$ has the interpretation of mass within a sphere of radius $r$, and $\dot{\mathcal{R}}=\partial \mathcal{R} / \partial t$. These spherically symmetric inhomogeneous dust models were first discovered by Lemaître [11] and further studied by Tolman [12] and Bondi [13]; see [14] for a detailed discussion.

It is a general result that in a spacetime with a metric of the form $-\mathrm{d} t^{2}+g_{i j}\left(t, x^{k}\right) \mathrm{d} x^{i} \mathrm{~d} x^{j}$, any test particle that is at rest in space follows a geodesic [2]. Therefore, the fundamental comoving observers in the LTB model follow geodesics. Moreover, each fundamental observer carries the standard orthonormal tetrad frame (discussed in the previous section) that has its axes along the directions of the coordinates employed in (32). It can be shown explicitly that such a tetrad frame is parallel transported along the geodesic path of a fundamental observer. The off-centre cosmological measurements of such observers have been discussed in [15]. We are interested, however, in the local tidal dynamics of nearby test particles.

It proves convenient, for the sake of simplicity, to orient the spatial axes of the Fermi frame such that the $X$-axis points along the polar $(\theta)$ direction, the $Y$-axis points along the azimuthal $(\phi)$ direction, and the $Z$-axis points along the radial $(r)$ direction, that is, for the purposes of this section we choose $\lambda^{\mu}{ }_{(0)}=u^{\mu}=\delta^{\mu}{ }_{0}, \lambda^{\mu}{ }_{(1)}=(0,0,1 / \mathcal{R}, 0), \lambda^{\mu}{ }_{(2)}=(0,0,0,1 /(\mathcal{R} \sin \theta))$ and $\lambda^{\mu}{ }_{(3)}=(0,1 / b, 0,0)$. We find that for a fundamental observer at a fixed position in space, the nonzero components of the curvature tensor can be obtained from

$$
\begin{aligned}
& { }^{F} R_{0101}={ }^{F} R_{0202}=K_{1}(T), \\
& { }^{F} R_{0303}=K_{2}(T), \\
& { }^{F} R_{3131}={ }^{F} R_{3232}=K_{3}(T), \\
& { }^{F} R_{1212}=K_{4}(T) .
\end{aligned}
$$

Thus the analogue of (16) in this case is given by $E=\operatorname{diag}\left(K_{1}, K_{1}, K_{2}\right), B=0$ and $N=$ $\operatorname{diag}\left(K_{3}, K_{3}, K_{4}\right)$. Here,

$$
\begin{aligned}
K_{1}(T) & =-\frac{\ddot{\mathcal{R}}}{\mathcal{R}}, & K_{4}(T) & =\frac{2}{\mathcal{R}^{2}}\left(\frac{1}{2} \dot{\mathcal{R}}^{2}-\mathcal{E}\right), \\
K_{2}(T) & =-\frac{\ddot{\mathcal{R}}^{\prime}}{\mathcal{R}^{\prime}}, & K_{3}(T) & =\frac{1}{\mathcal{R} \mathcal{R}^{\prime}} \frac{\partial}{\partial r}\left(\frac{1}{2} \dot{\mathcal{R}}^{2}-\mathcal{E}\right),
\end{aligned}
$$

where $t=T$ and $r$ is simply a constant in the final expressions. Then (1)-(3) together with (28) imply that the Fermi system $(T, \boldsymbol{X})$ along the worldline of any fundamental observer has a metric of the form

$$
\begin{aligned}
-\mathrm{d} s^{2}=-[1+ & \left.\left(K_{1} \sin ^{2} \Theta+K_{2} \cos ^{2} \Theta\right) \rho^{2}\right] \mathrm{d} T^{2}+\mathrm{d} \rho^{2}+\left(1-\frac{1}{3} K_{3} \rho^{2}\right) \rho^{2} \mathrm{~d} \Theta^{2} \\
+ & {\left[1-\frac{1}{3}\left(K_{4} \sin ^{2} \Theta+K_{3} \cos ^{2} \Theta\right) \rho^{2}\right] \rho^{2} \sin ^{2} \Theta \mathrm{d} \Phi^{2}, }
\end{aligned}
$$

which is axially symmetric about the $Z$-axis (i.e. the radial $r$-direction), as expected. Local dynamics in this spacetime is considered in the appendix.

We are particularly interested in the form of this metric in the neighbourhood of the fundamental observer at the centre of spherical symmetry $(r=0)$. Assuming that the spacetime manifold is smooth at $r=0$ [13], we can write

$$
\begin{aligned}
& \mathcal{R}(t, r)=r S(t)\left[1+\frac{1}{2} \Delta(t) r+\frac{1}{6} \Sigma(t) r^{2}+O\left(r^{3}\right)\right], \\
& \mathcal{E}(r)=\frac{1}{2} \mathcal{E}^{\prime \prime}(0) r^{2}+O\left(r^{3}\right)
\end{aligned}
$$




$$
M(r)=\frac{1}{6} M^{\prime \prime \prime}(0) r^{3}+O\left(r^{4}\right) .
$$

Here, the scale factor is given by $S(t)=\mathcal{R}^{\prime}(t, 0)>0$ and

$$
\Delta(t) \equiv \frac{\mathcal{R}^{\prime \prime}(t, 0)}{\mathcal{R}^{\prime}(t, 0)}, \quad \Sigma(t) \equiv \frac{\mathcal{R}^{\prime \prime \prime}(t, 0)}{\mathcal{R}^{\prime}(t, 0)} .
$$

Using these expressions, we find that

$$
\begin{aligned}
K_{1} & =\mathcal{U}(T)\left[1+\frac{1}{2} \eta r+O\left(r^{2}\right)\right], & & K_{2}=\mathcal{U}(T)\left[1+\eta r+O\left(r^{2}\right)\right], \\
K_{3} & =\mathcal{W}(T)[1+O(r)], & & K_{4}=\mathcal{W}(T)[1+O(r)],
\end{aligned}
$$

where

$$
\mathcal{U}=-\frac{\ddot{S}}{S}, \quad \mathcal{W}=\left(\frac{\dot{S}}{S}\right)^{2}-\frac{\mathcal{E}^{\prime \prime}(0)}{S^{2}},
$$

and $\eta=(2 \dot{S} \dot{\Delta}+S \ddot{\Delta}) / \ddot{S}$. The Fermi metric for $r=0$ then takes the form

$-\mathrm{d} s^{2}=-\left(1+\mathcal{U} \rho^{2}\right) \mathrm{d} T^{2}+\mathrm{d} \rho^{2}+\left(1-\frac{1}{3} \mathcal{W} \rho^{2}\right) \rho^{2}\left(\mathrm{~d} \Theta^{2}+\sin ^{2} \Theta \mathrm{d} \Phi^{2}\right)$.

It is now a simple matter to recognize that the gravitational field equations for metric (65) result in the analogues of equations (39) and (40) in this pressure-free case, namely,

$$
\begin{aligned}
& \mathcal{U}=\frac{4 \pi G}{3} \mu(t, 0)-\frac{\Lambda}{3}, \\
& \mathcal{W}=\frac{8 \pi G}{3} \mu(t, 0)+\frac{\Lambda}{3} .
\end{aligned}
$$

Here $\mu(t, 0)$ can be expressed as

$$
\mu(t, 0)=\frac{M^{\prime \prime \prime}(0)}{8 \pi} \frac{1}{S^{3}(t)}
$$

by virtue of equation (50).

It follows from a comparison of (64) with (18) that $-\mathcal{E}^{\prime \prime}(0)$ plays the role of spatial curvature in these inhomogeneous models. Moreover, $k(T)=q H^{2}$ in this case and it is interesting to compare this appearance of the deceleration parameter, defined in terms of $S(t)$ in the standard manner, with the effective deceleration parameter $Q$ obtained from the luminosity distance-redshift relation. It has been shown [10] that in the general pressure-free case,

$$
d_{L}=\frac{1}{H} z+\frac{1}{2 H}(1-Q) z^{2}+\cdots,
$$

where the inhomogeneity parameter is given by

$$
Q-q=\frac{1}{S H^{2}} \frac{\mathrm{d} \Delta(t)}{\mathrm{d} t} .
$$

The LTB models have recently received much attention as simple alternatives to the darkenergy models of the universe; see, for example, [9, 16-20] and references therein.

Equations (64) and (66)-(68) may be written as

$$
\frac{1}{2} \mathcal{E}^{\prime \prime}(0)=\frac{1}{2}\left(\frac{\mathrm{d} S}{\mathrm{~d} t}\right)^{2}-\frac{1}{6}\left[\frac{G M^{\prime \prime \prime}(0)}{S}+\Lambda S^{2}\right]
$$




$$
2 q H^{2}=H^{2}-\left[\frac{\mathcal{E}^{\prime \prime}(0)}{S^{2}}+\Lambda\right] .
$$

The scale factor $S(t)$ can be simply determined from (71) by quadratures. In general, however, a simple analytic solution is not available. The qualitative behaviour of $S(t)$ can be studied using the interpretation of (71) in terms of the radial motion of a 'particle' of unit mass with kinetic energy $\dot{S}^{2} / 2$ and total energy $\mathcal{E}^{\prime \prime}(0) / 2$. That is, a graph of the potential energy in (71) versus $S$ can be used to illustrate the fact that motion can take place only in regions where

$$
\mathcal{E}^{\prime \prime}(0)>-\frac{1}{3}\left[\frac{G M^{\prime \prime \prime}(0)}{S}+\Lambda S^{2}\right] .
$$

Alternatively, (71) can be integrated numerically with the initial condition that at the present epoch $t_{0}, S\left(t_{0}\right)=R_{0}$, where $R_{0}$ is the (spatial) curvature radius. Let us note that for $\mathcal{E}^{\prime \prime}(0)=0$ and $\Lambda=0$,

$$
S=\left[\frac{3}{4} G M^{\prime \prime \prime}(0) t^{2}\right]^{1 / 3},
$$

so that $H=2 /(3 t)$, and $q=1 / 2$ follows immediately from (72); therefore, this case corresponds to the familiar Einstein-de Sitter model. Equation (72) is used in the following section to estimate the influence of the cosmic gravitational field on the dynamics of the solar system.

\section{Local dynamics}

The expanding universe is over ten billion years old; therefore, the cosmic tidal acceleration within the solar system is expected to be relatively very small. The long-term cosmological evolution of an 'isolated' gravitationally bound system in an expanding universe is beyond the scope of this work; instead, we are interested in observable cosmological perturbations on the dynamics of the solar system. As explained in detail in the appendix, the influence of spatial inhomogeneities on a Keplerian binary system can be studied on the basis of the Fermi coordinate system (57) associated with the LTB model. However, the small anisotropy of the cosmic microwave background radiation implies that a useful estimate of the effect can be obtained by ignoring any deviations from spherical symmetry. Therefore, we consider the quasi-inertial Fermi coordinate system that can be established along the worldline of the fundamental observer at $r=0$. Within this coordinate system associated with equation (65), imagine the approximately elliptical relative orbit of a Keplerian binary system that is perturbed by the cosmic tidal acceleration

$$
\frac{\mathrm{d}^{2} \boldsymbol{X}}{\mathrm{d} T^{2}}+\frac{G M_{0}}{\rho^{3}} \boldsymbol{X}=\boldsymbol{g}_{\text {cosmos }}
$$

where at the present epoch, $\boldsymbol{g}_{\text {cosmos }}=-q_{0} H_{0}^{2} \boldsymbol{X}$ according to equation (65). Here, $M_{0}$ is the net inertial mass of the binary system. Let us note that if the members of the Keplerian binary were test particles, i.e. $M_{0} \rightarrow 0$, then equation (75) would simply reduce to the Jacobi equation at the present epoch, since the relative speed of the particles is assumed to be negligible compared to the speed of light; therefore, (75) originates from a superposition of weak gravitational forces. If the external tidal acceleration is turned off at any instant of time, the resulting orbit is the osculating ellipse at that instant with eccentricity $e$ and semimajor axis $A$. Thus, the Newtonian orbital energy of the osculating ellipse per unit reduced mass is $-G M_{0} /(2 A)$. On the other hand, it follows from (75) that the rate of change of this orbital energy is given by $\boldsymbol{g}_{\text {cosmos }} \cdot \boldsymbol{V}$, so that

$$
\frac{G M_{0}}{2 A^{2}} \frac{\mathrm{d} A}{\mathrm{~d} T}=\boldsymbol{g}_{\text {cosmos }} \cdot \boldsymbol{V} \text {. }
$$


For the osculating ellipse,

$$
\boldsymbol{X} \cdot \boldsymbol{V}=\omega_{\mathrm{K}} A^{2} e \sqrt{1-e^{2}} \frac{\sin \varphi}{1+e \cos \varphi},
$$

where $\omega_{\mathrm{K}}=\left(G M_{0} / A^{3}\right)^{1 / 2}$ is the Keplerian frequency and $\varphi$ is an azimuthal angle of the orbit ('true anomaly'). Moreover, it follows from (72) that $2 q_{0}=1-\chi_{0}$, where $\chi_{0}$ is the present value of

$$
\chi=\frac{1}{H^{2}}\left[\frac{\mathcal{E}^{\prime \prime}(0)}{S^{2}}+\Lambda\right]
$$

Putting equations (76)-(78) together, we find that $\mathrm{d} A / \mathrm{d} T$ has the same periodicity as the orbit and its average over an orbit vanishes, that is

$$
\frac{\mathrm{d} A}{\mathrm{~d} T}=-\frac{H_{0}^{2} A e}{\omega_{\mathrm{K}}}\left(1-\chi_{0}\right) \frac{\sqrt{1-e^{2}} \sin \varphi}{1+e \cos \varphi} .
$$

This is in fact the Lagrange planetary equation for the semimajor axis of the osculating ellipse in this particular case. It turns out that-on average- the cosmological perturbations under consideration here cause a precession of the orbit in its plane, but leave the orbit otherwise unchanged. The appendix should be consulted for further details about the average behaviour of the orbit in this case.

For the orbit of the Earth around the Sun,

$$
\frac{H_{0}^{2} A e}{\omega_{\mathrm{K}}} \approx \frac{1}{4} \times 10^{-9} \mathrm{~cm} \mathrm{yr}^{-1}
$$

where $e \approx 0.02, A \approx 1.5 \times 10^{13} \mathrm{~cm}$ and $H_{0} \approx 70 \mathrm{~km} \mathrm{~s}^{-1} \mathrm{Mpc}^{-1}$. Even if-as a result of spatial inhomogeneities or a nonzero cosmological constant - the absolute magnitude of the deceleration parameter is enhanced by several orders of magnitude or so, the rate of variation of the astronomical unit would still be too small to be detectable at present. This should be contrasted with the recent reported secular increase of the astronomical unit, based on radiometric data, amounting to about $10 \mathrm{~cm}$ per year [21, 22]. We therefore conclude that the presence of cosmological inhomogeneities (or a cosmological constant) cannot change the conclusion that the expansion of the universe has a negligible influence on the dynamics of the solar system.

The results of this section as well as the appendix indicate that solar-system anomalies [22] — such as the Pioneer anomaly_cannot be explained in terms of cosmological perturbations based upon the general relativistic cosmological models considered in the present work. This is particularly evident from the negligibly small magnitude of $\boldsymbol{g}_{\text {cosmos }}$ over the solar system, since for $|\boldsymbol{X}| \sim 100 \mathrm{AU}, H_{0}^{2}|\boldsymbol{X}| \sim 10^{-20} \mathrm{~cm} \mathrm{~s}^{-2}$. This is about thirteen orders of magnitude smaller than the anomalous acceleration of Pioneer spacecraft.

\section{Discussion}

For local systems, such as the solar system, each body is subject to the gravitational influence of the whole mass-energy content of the universe; therefore, the relative motion of bodies is only affected by the tidal acceleration of the cosmic gravitational field. The main purpose of this paper has been to study the general features of tidal dynamics in certain simple spherically symmetric cosmological models. Particular emphasis has been placed on inhomogeneous models, since spatial inhomogeneities can mimic dark energy in the luminosity distanceredshift relation; moreover, inhomogeneous cosmological models are of current interest as possible alternatives to the dark-energy models [9, 16-20]. Indeed, we have elucidated the 
influence of inhomogeneities on tidal dynamics in the simple models that we have considered. The results of this work could therefore be of interest in the theoretical study of the tidal evolution of galaxies.

The tidal influence of the cosmic gravitational field on the solar system can be estimated and the result turns out to be too small to be measurable in the foreseeable future. Specifically, the influence of the cosmic tidal field on local dynamics has been studied here with a view towards observable effects in the solar system. We have therefore considered in detail how a Keplerian binary system might be affected by the main cosmic tidal acceleration. The change in the semimajor axis as well as the 'slow' evolution of the elements of the osculating ellipse appears to be too small to be detectable at present for the cosmological models under consideration here. These results make it highly improbable that solar system anomalies that have recently received attention [22] could be of cosmological origin.

\section{Appendix. Local dynamics in LTB spacetime}

Imagine a Keplerian binary system consisting of masses $m_{1}$ and $m_{2}$ with positions $\boldsymbol{X}_{1}$ and $\boldsymbol{X}_{2}$ within the Fermi coordinate system associated with the LTB metric (57). It turns out that for linear tidal perturbations, the motion of the binary system can be simplified, essentially as in the standard unperturbed Kepler system, by a separation into the motion of the centre of mass and the relative motion; in this paper, we ignore the motion of the centre of mass of the perturbed binary and concentrate on the directly observable relative motion. The 'Newtonian' tidal potential

$$
\mathcal{V}_{\mathrm{N}}=\frac{1}{2} K_{1}(T)\left(X^{2}+Y^{2}\right)+\frac{1}{2} K_{2}(T) Z^{2}
$$

is quadratic in $\boldsymbol{X}$, so that the corresponding tidal acceleration, $-\nabla \mathcal{V}_{\mathrm{N}}$, is linear in $\boldsymbol{X}$. This implies that the external cosmological perturbation on the relative motion has the form

$$
\boldsymbol{F}=-K_{1}^{0} \boldsymbol{X}+\left(K_{1}^{0}-K_{2}^{0}\right) Z \hat{Z},
$$

where $\boldsymbol{X}=\boldsymbol{X}_{1}-\boldsymbol{X}_{2}$ describes relative position. Here, $K_{1}^{0}=K_{1}\left(T_{0}\right)$ and $K_{2}^{0}=K_{2}\left(T_{0}\right)$ are evaluated at the present epoch $T_{0}$. It is important to emphasize that our task here is to study the main observable consequences of cosmological perturbations on local systems; therefore, the external perturbation on the binary system is evaluated at the present epoch in equation (A.2). The equation of relative motion now takes the standard form

$$
\frac{\mathrm{d}^{2} \boldsymbol{X}}{\mathrm{d} T^{2}}+\frac{G M_{0} \boldsymbol{X}}{\rho^{3}}=\boldsymbol{F}
$$

where $M_{0}=m_{1}+m_{2}$. We assume that the binary system experiences small perturbations due to the tidal influence of the cosmological gravitational field. The perturbed Kepler system (A.3) can thus be treated using standard methods of celestial mechanics [23]. In our approach, the study of the long-term cosmological evolution of the Keplerian binary should be based upon the solution of (A.3) in the more general case in which $K_{1}(T)$ and $K_{2}(T)$ in $\boldsymbol{F}$ are not restricted to the present epoch; however, such an analysis is beyond the scope of this paper. On the other hand, the influence of the expansion of the universe on the binary system during the period of time that the orbit is monitored can be included in our perturbative treatment via Taylor expansions of $K_{1}(T)$ and $K_{2}(T)$ about $K_{1}^{0}$ and $K_{2}^{0}$, respectively, in powers of $T-T_{0}$ such that $\left|T-T_{0}\right| \ll T_{0}$. The first-order terms would have an even smaller effect on the relative orbit in comparison with the considerations of section 5; moreover, the results of this approach can be compared with previous work in this direction $[6,7]$.

The state of relative motion in (A.3) is given by the position and velocity at a given time $T$; on the other hand, one could employ instead the six orbital elements of the instantaneous 
osculating ellipse to specify the motion. The temporal evolution of these orbital elements is described by the Lagrange planetary equations [23], which are therefore equivalent to (A.3). For the orbital parameters, it is useful to employ Delaunay's action-angle elements $(\tilde{L}, \tilde{G}, \tilde{H}, \tilde{l}, \tilde{g}, \tilde{h})$ given by

$\tilde{L}=A^{1 / 2}, \quad \tilde{G}=\left[G M_{0} A\left(1-e^{2}\right)\right]^{1 / 2}, \quad \tilde{H}=\tilde{G} \cos i$,

$\tilde{l}=\vartheta-e \sin \vartheta, \quad \tilde{g}=$ argument of the pericentre, $\quad \tilde{h}=$ longitude of the ascending node.

Here, $A$ is the semimajor axis of the osculating ellipse, $e$ is its eccentricity, $i$ is the orbital inclination, $\vartheta$ is the eccentric anomaly and $\tilde{l}$ is the mean anomaly. Moreover, $\tilde{G}$ is the magnitude of the orbital angular momentum vector and $\tilde{H}$ is its $Z$-component. Along the osculating ellipse, the radial position $\rho$ can be expressed in terms of the true anomaly $\varphi$ and eccentric anomaly $\vartheta$, respectively, as follows:

$$
\rho=\frac{A\left(1-e^{2}\right)}{1+e \cos \varphi}, \quad \rho=A(1-e \cos \vartheta) .
$$

In equation (A.4) and throughout, we consider only positive square roots. To express the dynamical equations in terms of Delaunay's elements, it is necessary to decompose the perturbing acceleration $\boldsymbol{F}$ in terms of an orthonormal frame field adapted to the osculating ellipse. That is, we can write

$$
\boldsymbol{F}=F_{\rho} \hat{\boldsymbol{\rho}}+F_{s} \hat{\boldsymbol{s}}+F_{n} \hat{\boldsymbol{n}},
$$

in terms of its radial, sideways and normal components. Here $\hat{\rho}=\boldsymbol{X} / \rho$ is the radial unit vector, $\hat{s}=\hat{\boldsymbol{n}} \times \hat{\boldsymbol{\rho}}$ and $\hat{\boldsymbol{n}}$ is the unit vector in the direction of the orbital angular momentum $\tilde{G}$; that is, $\tilde{\boldsymbol{G}}=\tilde{G} \hat{\boldsymbol{n}}$. Thus, $\hat{\boldsymbol{\rho}}$ and $\hat{s}$ are in the instantaneous orbital plane of the osculating ellipse, while $\hat{\boldsymbol{n}}$ is normal to it.

The equations of motion in terms of Delaunay's elements are then given by

$$
\begin{aligned}
\frac{\mathrm{d} \tilde{L}}{\mathrm{~d} T} & =\frac{\tilde{L}^{3}}{\tilde{G}}\left[F_{\rho} e \sin \varphi+F_{s}(1+e \cos \varphi)\right], \\
\frac{\mathrm{d} \tilde{G}}{\mathrm{~d} T} & =\rho F_{s}, \\
\frac{\mathrm{d} \tilde{H}}{\mathrm{~d} T} & =\rho\left[F_{s} \cos i-F_{n} \sin i \cos (\varphi+\tilde{g})\right], \\
\frac{\mathrm{d} \tilde{l}}{\mathrm{~d} T} & =\omega_{\mathrm{K}}+\frac{\rho}{\omega_{\mathrm{K}} A^{2} e}\left[F_{\rho}\left(-2 e+\cos \varphi+e \cos ^{2} \varphi\right)-F_{s}(2+e \cos \varphi) \sin \varphi\right], \\
\frac{\mathrm{d} \tilde{g}}{\mathrm{~d} T} & =-\frac{\rho F_{n}}{\tilde{G}} \frac{\cos i}{\sin i} \sin (\varphi+\tilde{g})+\frac{\left(1-e^{2}\right)^{1 / 2}}{\omega_{\mathrm{K}} A e}\left(-F_{\rho} \cos \varphi+F_{s} \frac{2+e \cos \varphi}{1+e \cos \varphi} \sin \varphi\right), \\
\frac{\mathrm{d} \tilde{h}}{\mathrm{~d} T} & =\frac{\rho F_{n}}{\tilde{G}} \frac{\sin (\varphi+\tilde{g})}{\sin i},
\end{aligned}
$$

where $\omega_{\mathrm{K}}=\left(G M_{0}\right)^{1 / 2} / \tilde{L}^{3}$. A direct derivation of these equations is essentially contained in appendix B of [24].

To find the explicit form of $F_{\rho}, F_{s}$ and $F_{n}$ in terms of Delaunay's elements, we note that 


$$
\begin{aligned}
X & =\rho[\cos \tilde{h} \cos (\varphi+\tilde{g})-\sin \tilde{h} \cos i \sin (\varphi+\tilde{g})] \\
Y & =\rho[\sin \tilde{h} \cos (\varphi+\tilde{g})+\cos \tilde{h} \cos i \sin (\varphi+\tilde{g})], \\
Z & =\rho \sin i \sin (\varphi+\tilde{g}) .
\end{aligned}
$$

Moreover [24],

$$
\begin{aligned}
\hat{\boldsymbol{n}}= & (\sin \tilde{h} \sin i,-\cos \tilde{h} \sin i, \cos i), \\
\hat{\boldsymbol{s}}= & (-\cos \tilde{h} \sin (\varphi+\tilde{g})-\sin \tilde{h} \cos i \cos (\varphi+\tilde{g}), \\
& -\sin \tilde{h} \sin (\varphi+\tilde{g})+\cos \tilde{h} \cos i \cos (\varphi+\tilde{g}), \sin i \cos (\varphi+\tilde{g})) .
\end{aligned}
$$

A straightforward calculation using (A.2) reveals that

$$
\begin{aligned}
& F_{\rho}=-K_{1}^{0} \rho+\left(K_{1}^{0}-K_{2}^{0}\right) \rho \sin ^{2} i \sin ^{2}(\varphi+\tilde{g}), \\
& F_{s}=\left(K_{1}^{0}-K_{2}^{0}\right) \rho \sin ^{2} i \sin (\varphi+\tilde{g}) \cos (\varphi+\tilde{g}), \\
& F_{n}=\left(K_{1}^{0}-K_{2}^{0}\right) \rho \sin i \cos i \sin (\varphi+\tilde{g}) .
\end{aligned}
$$

Substituting these results in equations (A.8)-(A.13) and averaging over the 'fast' orbital motion, one can determine the 'slow' evolution of the orbit under cosmological perturbations in this case.

The weak external perturbation (A.2) naturally splits into two terms: one that is proportional to $K_{1}^{0}$ and the other proportional to $K_{1}^{0}-K_{2}^{0}$. The influence of these on the orbit can be analysed separately and the results can be superimposed in accordance with our linear perturbation scheme. Let us therefore first consider a tidal perturbation of the form $-K_{1}^{0} \boldsymbol{X}$. It turns out that this problem has already been solved in a different context [25]; in fact, the details of the averaging procedure are given in section IV of [25] for a perturbing acceleration of the form $\lambda \boldsymbol{X}$, where $\lambda$ is a constant. It is interesting to note that in [25], $\lambda=\Lambda c^{2} / 3$, where $\Lambda$ is the cosmological constant associated with the Kerr-de Sitter spacetime. It is shown in [25] that the orbit is planar and its semimajor axis and eccentricity do not change on average; however, the pericentre precesses with frequency $3 \lambda\left(1-e^{2}\right)^{1 / 2} \hat{\boldsymbol{n}} /\left(2 \omega_{\mathrm{K}}\right)$. Thus the net average effect of the first term in (A.2) on the orbit is to generate a pericentre precession of frequency $-3 K_{1}^{0}\left(1-e^{2}\right)^{1 / 2} \hat{\boldsymbol{n}} /\left(2 \omega_{\mathrm{K}}\right)$.

The second term in (A.2) is a perturbing acceleration in the $Z$-direction, which corresponds to the radial direction in the standard form of the LTB spacetime. Inspection of equations (A.8)-(A.13) reveals that $\mathrm{d} \tilde{H} / \mathrm{d} T=0$, hence the $Z$-component of the orbital angular momentum remains unchanged; moreover, there is clearly no effect to linear order if the orbit lies in the $(X, Y)$-plane. Hence we assume $i \neq 0$. It is then straightforward to average the right-hand sides of the remaining equations over the 'fast' motion with frequency $\omega_{\mathrm{K}}=2 \pi / T_{\mathrm{K}}$ such that for a function $\mathcal{F},\langle\mathcal{F}\rangle=T_{\mathrm{K}}^{-1} \int_{0}^{T_{\mathrm{K}}} \mathcal{F} \mathrm{d} t$, or equivalently,

$$
\langle\mathcal{F}\rangle=\frac{\left(1-e^{2}\right)^{3 / 2}}{2 \pi} \int_{0}^{2 \pi} \frac{\mathcal{F} \mathrm{d} \varphi}{(1+e \cos \varphi)^{2}},
$$

using the unperturbed orbit. The resulting integrals can be evaluated in principle, but it is simpler to express the main results for a slightly eccentric orbit. We find that

$$
\left\langle\frac{\mathrm{d} A}{\mathrm{~d} T}\right\rangle=O\left(e^{2}\right), \quad\left\langle\frac{\mathrm{d} e}{\mathrm{~d} T}\right\rangle=O(e),
$$




$$
\begin{aligned}
\left\langle\frac{\mathrm{d} \tilde{g}}{\mathrm{~d} T}\right\rangle & =-\frac{\left(K_{1}^{0}-K_{2}^{0}\right) \cos ^{2} i}{2 \omega_{\mathrm{K}}}+O(e), \\
\left\langle\frac{\mathrm{d} \tilde{h}}{\mathrm{~d} T}\right\rangle & =\frac{\left(K_{1}^{0}-K_{2}^{0}\right) \cos i}{2 \omega_{\mathrm{K}}}+O\left(e^{2}\right) .
\end{aligned}
$$

Thus the main effects here are the precessions of the pericentre and the ascending node with frequencies given by (A.24)-(A.25). The net average effect of (A.2) on the orbit is mainly the precession of the orbit in its own plane as well as about the $Z$-axis (i.e. the LTB radial direction); the latter motion occurs with frequency $\left(K_{1}^{0}-K_{2}^{0}\right) \cos i /\left(2 \omega_{\mathrm{K}}\right)$.

It should be noted that a discussion of observable relativistic effects would be incomplete without a proper treatment of the methods that could be employed for the measurement of such effects. For instance, such methods may involve light signals; therefore, in the case under consideration, the propagation of electromagnetic radiation in the cosmic tidal field may have to be taken into account. However, the local deviation of the cosmological Fermi metric from the flat background Minkowski metric involves dimensionless tidal potentials that are extremely small compared to unity; for example, we note that $H_{0}^{2} \rho^{2} /\left(2 c^{2}\right) \sim 10^{-30}$ for $\rho \sim 1$ AU. Thus the net influence of cosmology on local measurement techniques is expected to be negligibly small in this case.

Finally, let us restrict our general off-centre LTB treatment to a situation where the fundamental observer is very close to the centre of spherical symmetry. Indeed, the maximum anisotropy in the temperature of the cosmic background radiation is about 0.002 due mainly to our peculiar motion relative to this radiation bath; therefore, within the LTB model a reasonable estimate may be obtained by assuming $r=0$ as in section 5. Indeed, equation (62) implies that $K_{1}-K_{2} \rightarrow 0$ for $r \rightarrow 0$. It follows that the cosmological perturbations on average leave the shape of the orbit and the orientation of the orbital plane unchanged, but cause a pericentre precession of frequency $-3 q_{0} H_{0}^{2}\left(1-e^{2}\right)^{1 / 2} \hat{\boldsymbol{n}} /\left(2 \omega_{\mathrm{K}}\right)$. For the motion of the Earth around the Sun, $H_{0}^{2} / \omega_{\mathrm{K}} \approx 10^{-21} / \mathrm{yr}$, which is some fourteen orders of magnitude smaller than the Einstein pericentre precession. Thus the cosmological pericentre precession does not appear to be detectable in the foreseeable future. This conclusion applies to all of the cosmological models considered in this paper, so long as any deviation from isotropy about the Keplerian system can be neglected.

\section{References}

[1] Synge J L 1960 Relativity: The General Theory (Amsterdam: North-Holland) Manasse F K and Misner C W 1963 J. Math. Phys. 4735 Mashhoon B 1977 Astrophys. J. 216591

[2] Chicone C and Mashhoon B 2002 Class. Quantum Grav. 194231 Chicone C and Mashhoon B 2004 Class. Quantum Grav. 21 L139 Chicone C and Mashhoon B 2005 Class. Quantum Grav. 22195

[3] Chicone C, Mashhoon B and Punsly B 2004 Int. J. Mod. Phys. D 13945 Chicone C, Mashhoon B and Punsly B 2005 Phys. Lett. A 3431 Chicone C and Mashhoon B 2005 Ann. Phys., Leipzig 14290 Chicone C and Mashhoon B 2005 Astron. Astrophys. 437 L39

[4] Chicone C and Mashhoon B 2006 Phys. Rev. D 74064019

[5] Mashhoon B 1984 The Big Bang and Georges Lemaître ed A Berger (Dordrecht: Reidel) pp 75-81

[6] Cooperstock F I, Faraoni V and Vollick D N 1998 Astrophys. J. 50361 Bonnor W B 2000 Gen. Rel. Grav. 321005 Mizony M and Lachièze-Rey M 2005 Astron. \& Astrophys. 43445 Lämmerzahl C, Preuss O and Dittus H 2007 Lasers, Clocks, and Drag-Free Control: Exploration of Relativistic Gravity in Space ed H Dittus, C Lämmerzahl and S G Turyshev (Berlin: Springer) (Preprint gr-qc/0604052) 
Sereno M and Jetzer P 2007 Phys. Rev. D 75064031

Adkins G S, McDonnell J and Fell R N 2007 Phys. Rev. D 75064011

Faraoni V and Jacques A 2007 Preprint arXiv:0707.1350

[7] Krasiński A 1997 Inhomogeneous Cosmological Models (Cambridge: Cambridge University Press)

[8] Célérier M N 2000 Astron. Astrophys. 35363

Dabrowski M P and Hendry M A 1998 Astrophys. J. 49867

Pascual-Sánchez J F 1999 Mod. Phys. Lett. A 141539

Tomita K 2000 Astrophys. J. $\mathbf{5 2 9} 38$

[9] Célérier M N 2007 New Adv. Phys. 129

[10] Partovi M H and Mashhoon B 1984 Astrophys. J. 2764

[11] Lemaître G 1933 Ann. Soc. Sci. Brux. A 5351

Lemaître G 1997 Gen. Rel. Grav. 29637 reprinted (Engl. transl.)

[12] Tolman R C 1934 Proc. Natl Acad. Sci. USA 20169

Tolman R C 1997 Gen. Rel. Grav. 29931 (reprinted)

[13] Bondi H 1947 Mon. Not. R. Astron. Soc. 107410

Bondi H 1999 Gen. Rel. Grav. 311777 (reprinted)

[14] Plebański J and Krasiński A 2006 An Introduction to General Relativity and Cosmology (Cambridge: Cambridge University Press)

[15] Humphreys N P, Maartens R and Matravers D R 1997 Astrophys. J. 47747

[16] Iguchi H, Nakamura T and Nakao K 2002 Prog. Theor. Phys. 108809

[17] Mansouri R 2005 Preprint astro-ph/0512605, astro-ph/0601699

[18] Moffat J W 2006 J. Cosmol. Astropart. Phys. JCAP05(2006)001

[19] Apostolopoulos P S, Brouzakis N, Tetradis N and Tzavara E 2006 J. Cosmol. Astropart. Phys. JCAP06(2006)009

[20] Alnes H and Amarzguioui M 2007 Phys. Rev. D 75023506

[21] Krasinsky G A and Brumberg V A 2004 Celest. Mech. Dyn. Astron. 90267

Standish E M 2005 Proceedings of the IAU Colloquium No. 196, ed D W Kurtz (Cambridge: Cambridge University Press) pp 163-79

[22] Lämmerzahl C, Preuss O and Dittus H 2006 Preprint gr-qc/0604052

[23] Danby J M A 1988 Fundamentals of Celestial Mechanics 2nd edn (Richmond, VA: Willmann-Bell)

[24] Chicone C, Mashhoon B and Retzloff D G 1999 Class. Quantum Grav. 16507

[25] Kerr A W, Hauck J C and Mashhoon B 2003 Class. Quantum Grav. 202727 